\title{
Forward Calorimeters for the Future Electron-Positron Linear Collider Detectors
}

\section{O. Novgorodova ${ }^{1}$}

1. DESY, Zeuthen, Germany

E-mail: olga.novgorodova@desy.de

K.Afanaciev ${ }^{3}$, J.Aguilar ${ }^{5,6}$, H.Henschel ${ }^{1}$, M. Idzik ${ }^{6}$, A.Ignatenko ${ }^{7}$, Sz.Kulis ${ }^{6}$, S.Kollowa ${ }^{4}$, W.Lange ${ }^{1}$, I.Levy ${ }^{2}$, W.Lohmann ${ }^{1}$, S.Schuwalow ${ }^{1}$

2. Tel Aviv University, Tel Aviv, Israel

3. NCPHEP, Minsk, Belarus

4. BTU Cottbus, Cottbus, Germany

5. Institute of Nuclear Phyis, Krakow, Poland

6. AGH University of Science and Technology Department of Physic, Krakow, Poland

7. DESY, Hamburg, Germany

\begin{abstract}
In future electron-positron collider detectors, special calorimeters are needed in the very forward region; one of them, the Beam Calorimeter (BeamCal) is designed as a GaAs-Tungsten sandwich calorimeter. Several GaAs sensor sectors were tested in the laboratory. The current as a function of the voltage was measured for each pad and found to be about $100 \mathrm{nA}$ at $60 \mathrm{~V}$. One sensor sector was assembled with fan-out electronics bonded on one side to a sensor pad and on the other side to an front-end (FE) ASIC. The fully assembled system was then investigated in an electron beam. Preliminary results of the test beam data analysis are reported. Charge collection efficiency (CCE) and signal to noise were calculated form test beam data.
\end{abstract}

The XIXth International Workshop on High Energy Physics and Quantum Field Theory - QFTHEP2010 Golitsyno, Moscow, Russia September 08-15 2010

$1 \quad$ Speaker 


\section{Introduction}

The International Linear Collider, ILC, is one of the options for a future linear electron-positron collider. The high luminosity needed for the physics program result in very small beam sizes and high number of bunch crossings, what will create beamstrahlung. The beamstrahlung photons are emitted under a very low angle [1], but can be convert into electron-positron pairs which will hit the forward region of the detectors at the ILC and cause background for the inner detectors like Time Projection Chamber (TPC), Silicon pixel detector and the forward calorimeters.

In the forward region of the International Linear Detector (ILD) and Silicon Detector (SiD) detector concepts, special calorimeters will provide precise luminosity measurements Luminosity Calorimeter (LumiCal), identification of single high energy electrons (sHEe) Beam Calorimeter (BeamCal), beam diagnostics and luminosity monitoring.

The integrated luminosity will be determined by counting the total number of Bhabha events produced in the acceptance region of the LumiCal and the corresponding cross section, which should be calculated in QED to the required precision of less than $10^{-3}$ (studied in [2]). Additionally, the BeamCal will provide ILC with an instantaneous luminosity measurement. The BeamCal will be located behind LumiCal and Low-angle Hadron Calorimeter (LHCal), and will surround two crossing beam pipes. Due to high background from beamstrahlung photons at the ILC, BeamCal is expected to receive high doses of radiation at about $10 \mathrm{MGy}$ [3]. LumiCal and BeamCal are designed as 30-layer sensor-tungsten sandwich calorimeter. Each layer consists of a tungsten absorber plate of 1 radiation length thickness and a sensor plane. For LumiCal, silicon sensors, and BeamCal, GaAs sensors, are foreseen. All sensors have fine segmentation. The geometries of the calorimeters have been studied in [ 4, 5]. The first prototypes of LumiCal and BeamCal sensors were tested to prove operation of the full read-out chain of sensor planes with FE-electronics.

\section{BeamCal Sensor Plane}

Semi-insulating (SI) Liquid Encapsulated

Czochralski (LEC) GaAs is well known as a material for radiation detectors and was shown to be a good candidate for the BeamCal for the ILC [6]. We are investigating GaAs detector as a position-sensitive detectors. The produced GaAs sensor panes are made from a compensated high-ohmic Cr-doped GaAs material, and are doped with tin and tellurium as shallow donors and chromium as a deep acceptor. The tested GaAs sensors, as shown in Fig. 1, were produced in Tomsk, Russia. Several planes were tested in the laboratory. The one with the lowest leakage current was chosen to build a prototype. The leakage current of chosen sensor plane was measured to be about $100 \mathrm{nA}$ at $60 \mathrm{~V}$. Each plane forms oneeighth of a circular ring, with inner rad $2 \mathrm{~cm}$ and outer $\operatorname{rad} 8.5 \mathrm{~cm}$. The thickness of the sensor plane is 500 um. The sensor is segmented radially into 12 rings Each ring is composed of square pads of side length

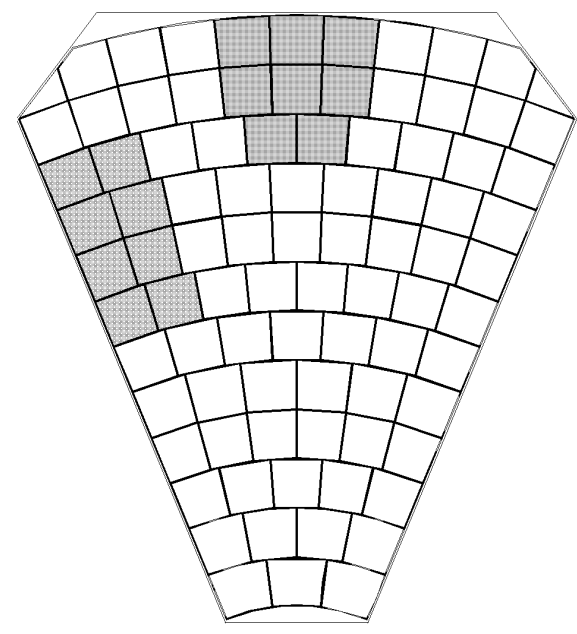

Fig. 1: Sensor plane segmentation with two regions under investigation 
$0.5 \mathrm{~cm}$. The prepared prototype consists of a sensor plate, read out electronics, front-end ASICS, fan-out and an ADC v1721 for digitizing signals.

\section{Experimental set up}

\subsection{Test beam DESY II}

The beam test was performed in the summer of 2010 at DESY-II area 22 [7, 8], using $4.5 \mathrm{GeV}$ electrons. DESY-II can provide electrons with energies up to $7 \mathrm{GeV} / \mathrm{c}$, with particle rates depending strongly on energy. The trigger rate used was around a few hundred hertz.

\subsection{ZEUS MVD telescope}

For the track measurement of each beam particle the ZEUS telescope was used. It consists of three modules and every module containing two perpendicular layers of silicon strip detectors (Reference detectors 1, 2 and 3). Hence, each module provides $\mathrm{x}$, $\mathrm{y}$ and $\mathrm{z}$ coordinates which can be used for track reconstruction to predict the hit position in the device under test (DUT). Each layer is a single-side silicon strip detector with $300 \mu \mathrm{m}$ thick and $32 \mathrm{~mm}$ x 32 $\mathrm{mm}$ area. The strip pitch of $25 \mu \mathrm{m}$ and a read-out pitch of $50 \mu \mathrm{m}$ [9].

\subsection{Device under test}

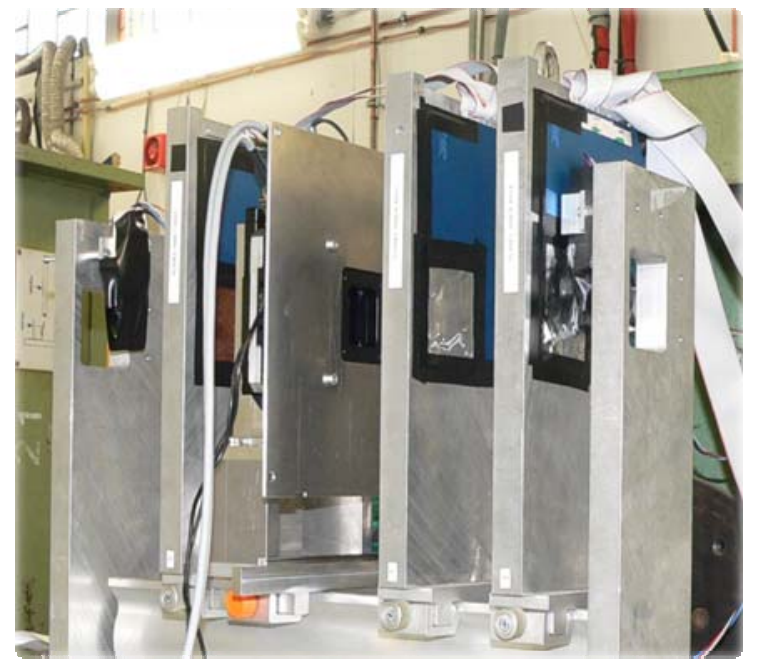

Fig. 2: The experimental set up: Trigger, telescope, xy-table, DUT

The DUT, or sensor box, was prepared before arrival at the beam test site. Main part of the sensor box is a printed circuit board, which provides the read-out electronics, power supplies and a HV filter. The read-out circuitry comprises preamplifier ASICs, developed by the Krakow group and line drivers for signal transmission over long coax cables. First the GaAs sensor was glued to the main board using conductive glue. Second, a thin PCB with fan-out traces was screwed to the main board on top of the sensor. Then the electrodes of the sensor pads were connected to the fan-out and fan-out traces on the front-end ASICs by wire bonding.

\subsection{Sensor plane}

To study the performance of the sensor plane as a function of position, the DUT was put between second and third planes. The DUT was set up on a remotely-controlled precision x-y table, which allowed the DUT to be moved in the beam by up to $4.8 \mathrm{~cm}$ each in the $\mathrm{x}$ - and $\mathrm{y}-$ directions. The x-y table itself was mounted on the same optical bench as the Si telescope. The modules were fixed in the following positions: the first telescope plane was taken as the reference zero position in the z-axis along the beam. The second telescope plane was located at 
$10 \mathrm{~cm}$, the $\mathrm{x}-\mathrm{y}$ table was mounted at $18.6 \mathrm{~cm}$ and the last telescope plane at $32.5 \mathrm{~cm}$ from the first plane. The system was operated at room temperature of about $27^{\circ} \mathrm{C}$.

Two data acquisition systems (DAQ) were used: one for the telescope DAQ [10] and one for DUT DAQ. The latter was adopted from the fast beam conditions monitor BCM1F for the CMS Experiment [11]. Coincidence of three scintillator fingers located at both ends of the optical bench was used to generate a trigger to start the DAQ systems. Time stamps were used to synchronize the events recorded by the DAQ systems.

\section{Measurements}

For the test beam several goals were planned. The first and most basic was to test the operation of the front-end electronics together with a sensor and automated readout. Second goal was to measure characteristics of the irradiated pads as a function of the impact point of beam electrons, in order to study the uniformity of charge collection efficiency near the pad edges. For this high statistics are needed since the area between pads is small. Another important parameter to investigate is crosstalk, and in particular its dependence on the length and position of the signal traces.

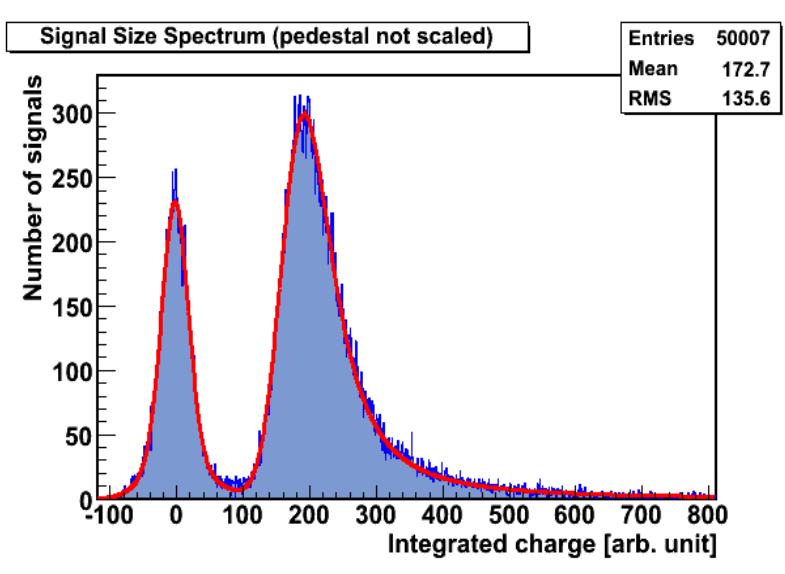

Fig. 3: Signal spectrum

\section{Analysis and tracks reconstruction}

For every trigger the signal in DUT is recorded using $500 \mathrm{~ms} 8$ bit flash ADC. These signals can be integrated over time for analysis. An example of spectra obtained using an integrating window of $400 \mathrm{~ns}$ can be seen on the Figure 3. The peak on the left side is the pedestal and the peak on the right results from a particle crossing the pad. The spectrum can be fitted by Gaussian and Landau distribution convoluted with a Gaussian for the signal. The difference between the most probable values of the signal and pedestal determine the CCE and $\mathrm{S} / \mathrm{N}$ ratio. The calculated CCE for all 16 irradiated pads is about $27.3 \pm 2.6 \%$. The $\mathrm{S} / \mathrm{N}$ ratio is about $8.6 \pm 1.7$.

For the track reconstruction and hit position estimation, only two telescope planes were taken into account. With two $\mathrm{x}, \mathrm{y}$ and $\mathrm{z}$ spatial positions, the hit position was calculated from the linear extrapolation to the DUT z-position.

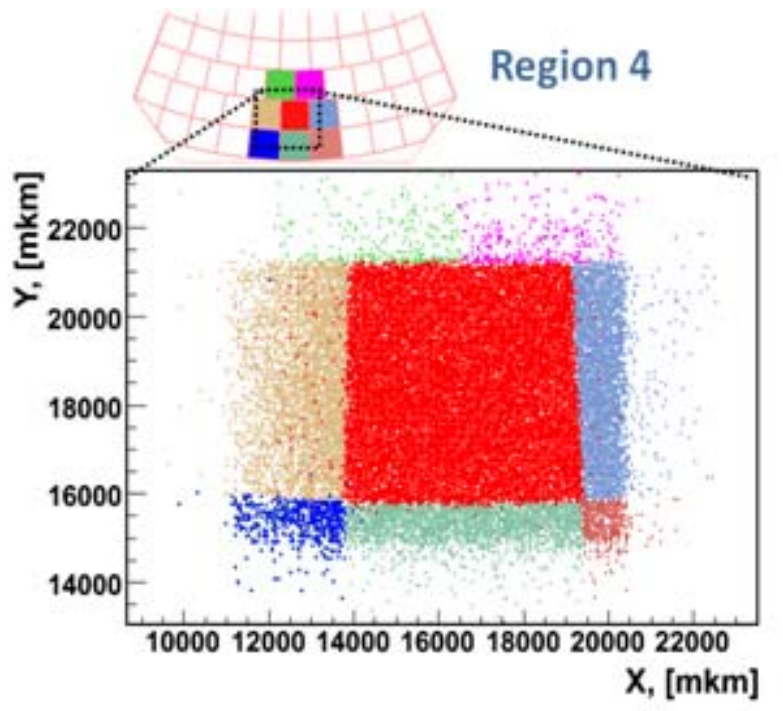

Fig. 4: The pad structure reconstruction for region 4 
For synchronization between data acquisition systems of telescope and DUT veto algorithm was used and with latest verification of synchronization. In Fig. 4 the structure of irradiated pads of one of the investigated regions is shown. For every pad, thresholds were set up and when signals were higher than one or another threshold, different colors were applied for pads. In Fig. 5 the number of hits as a function of the reconstructed x position on the pad is shown as an example of uniformity of beam particles distributed over chosen irradiated pad. High telescope resolution permits investigation of pad characteristics like CCE as a function of beam particle position. Preliminarily results with low statistics are shown on the Fig. 6. Every bin of this histogram represents chosen area of pad and collected signals spectra fitted with gauss for pedestal and landau-gauss convolution for the signals. The CCE was calculated as described in [12]. Obtained preliminarily results show CCE uniformity over whole pad.

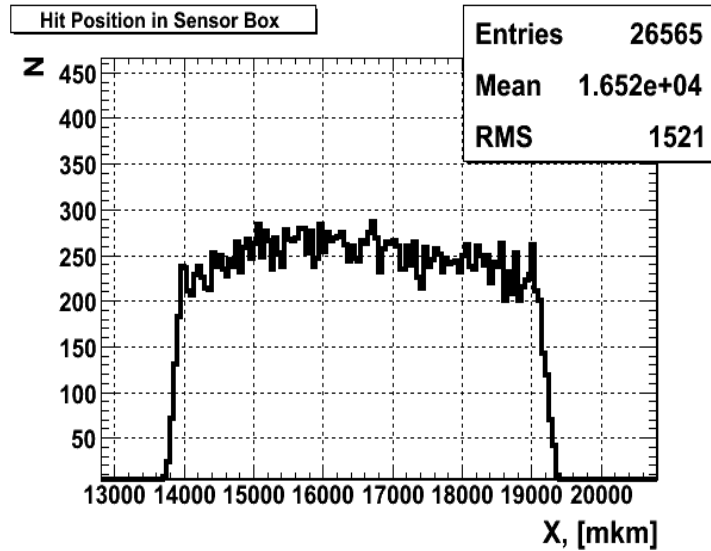

Fig. 5: Number of hits as a function of the reconstructed $\mathrm{x}$ position in sensor box.

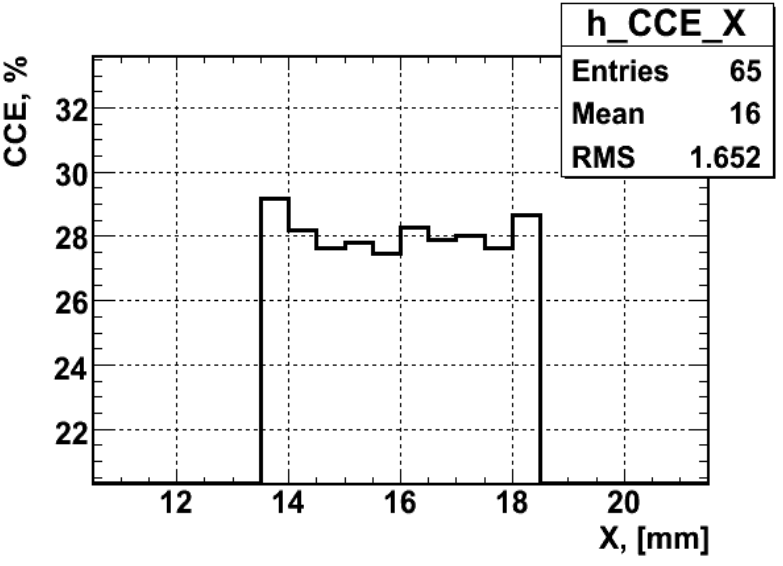

Fig. 6: CCE as a function of reconstructed $\mathrm{x}$ position in sensor box.

\section{Conclusions}

In the summer 2010, the first measurement combining a GaAs sensor with a front-end ASIC was done in the test beam DESYII (Hamburg). For 16 pads out of 85 pads I-V, C-V, CCE, S/N ratio, CCE vs position were measured. The results are very promising. Further work is needed for better understanding of obtained test beam data and for using full statistics.

\section{Acknowledgments}

The authors wish to thank I.-M. Gregor and A. Kravchenko for their help providing us with telescope and telescope analysis program. We are also grateful to DESY providing us test beam time. This work is supported by the 7th Framework Program "Marie Curie ITN", grant agreement number 214560. And we would like to thank the FP7 multi-site Initial Training Network on particle detectors in physics experiments and by EUDET for support. 


\section{References}

1. D Schulte, Study of electromagnetic and hadronic background in the interaction region of the TESLA collider, TESLA 97-08, 1996

2. Achim Stahl, Luminosity measurement via Bhabha scattering: precision requirements for the luminosity calorimeter, LC-DET-2005-004, 2005.

3. H. Abramowicz et al., Forward Instrumentation for ILC Detectors, Instrumentation and Detectors, arXiv:1009.2433v2, 3 Nov 2010

4. H. Abramowicz et al., Redefinition of the geometry of the luminosity calorimeter, EUDET-Memo-2008-09, 2008, http://www.eudet.org.

5. P. Bambade, V. Drugakov andW. Lohmann, The impact of Beamcal performance at different ILC beam parameters and crossing angles on stau searches, Pramana J. Phys. 69 (2007) 1123.

6. International Linearcollider Reference Report, http://www.linearcollider.org/about/Publications/Reference-Design-Report.

7. http://www.eudet.org

8. D.Autiero, P.Migliozzi et.al., OPERA Collaboration, Characterization of the T24 electron beam line available at DESY, March 12, 2004

9. http://www.desy.de/ gregor/MVD_Telescope/short_intro.html

10. http://www.desy.de/ gregor/short_intro.html

11. http://cdsweb.cern.ch/record/1222323

12. M. Ohlerich, Investigations of the physics potential and detector development for the ILC, http://opus.kobv.de/btu/volltexte/2010/1440/ 